\title{
Cooling by Time Reversal of Atomic Matter Waves
}

\author{
J. Martin, B. Georgeot, and D. L. Shepelyansky \\ Laboratoire de Physique Théorique, Université de Toulouse III, CNRS, 31062 Toulouse, France
}

(Received 25 October 2007; published 1 February 2008)

\begin{abstract}
We propose an experimental scheme which allows us to realized approximate time reversal of matter waves for ultracold atoms in the regime of quantum chaos. We show that a significant fraction of the atoms return back to their original state, being at the same time cooled down by several orders of magnitude. We give a theoretical description of this effect supported by extensive numerical simulations. The proposed scheme can be implemented with existing experimental setups.
\end{abstract}

DOI: 10.1103/PhysRevLett.100.044106

PACS numbers: 05.45.Mt, 03.75.-b, 37.10.De, 37.10.Vz

The statistical theory of gases developed by Boltzmann leads to macroscopic irreversibility and entropy growth even if dynamical equations of motion are time reversible. This contradiction was pointed out by Loschmidt and is now known as the Loschmidt paradox [1]. The reply of Boltzmann relied on the technical difficulty of velocity reversal for material particles [2]: a story tells that he simply said "then go and do it". The modern resolution of this famous dispute came with the development of the theory of dynamical chaos [3-5]. Indeed, for chaotic dynamics small perturbations grow exponentially with time, making the motion practically irreversible. This explanation is valid for classical dynamics, while the case of quantum dynamics requires special consideration. Indeed, in the quantum case the exponential growth takes place only during the rather short Ehrenfest time [6], and the quantum evolution remains stable and reversible in presence of small perturbations [7]. Quantum reversibility in presence of various perturbations has been actively studied in recent years and is now described through the Loschmidt echo (see [8] and references therein). This quantity measures the effect of perturbations and is characterized by the fidelity $f\left(t_{r}\right)=\left|\left\langle\psi_{p}\left(2 t_{r}\right) \mid \psi(0)\right\rangle\right|^{2}$, where $\left|\psi_{p}\right\rangle$ is the time reversed wave function in presence of perturbations, $|\psi\rangle$ is the unperturbed one, and $t_{r}$ is the moment of time reversal. Experimental implementations of time reversibility for quantum dynamics or propagating waves have been realized with spin systems (spin echo technique) [9], acoustic [10] and electromagnetic [11] waves, resulting in various technological applications. Surprisingly enough, the reversibility signal becomes stronger and more robust in the case of chaotic ray dynamics [10]. However, despite the significant experimental progress made recently in the control of quantum systems, the time reversal of matter waves has not been performed so far.

Here we present a concrete experimental proposal of an effective time reversal of atomic matter waves. The proposal relies on the kicked rotator model, which is a cornerstone model of quantum chaos $[6,7,12]$. This model has been built up experimentally with cold atoms in kicked optical lattices [13-16]. Recent progress allowed to imple- ment this model with ultracold atoms and Bose-Einstein condensates (BEC) [17-20], with high-precision subrecoil definition of the momentum of the atoms, allowing, for example, to observe [17,19] high order quantum resonances [21]. We show that these experimental techniques allow to perform time reversal for a significant part of the atoms. Surprisingly, this fraction of the atoms becomes cooled down by several orders of magnitude during the process. We call this new cooling mechanism Loschmidt cooling since it is directly related to the time reversibility.

The quantum kicked rotator corresponds to the quantization of the Chirikov standard map [4,5]:

$$
\bar{p}=p+k \sin x, \quad \bar{x}=x+T \bar{p},
$$

where $x$ is the position and $p$ the momentum of an atom, and bars denote the variables after one map iteration. Here $x$ is a continuous variable in the interval $]-\infty,+\infty[$. The physical process behind corresponds to rapid change of momentum created by a kick of optical lattice followed by a free propagation of the atoms between periodic kicks of period $T$. The classical dynamics depends only on the single parameter $K=k T$ and undergoes a transition from integrability to chaos when $K$ is increased. Global chaos sets in for $K>K_{c}=0.9716 \ldots$ The dynamics of (1) is time reversible, e.g., by inverting all velocities at the middle of the free motion between two kicks.

The quantum evolution over one period is described by a unitary operator $\hat{U}$ acting on a wave function:

$$
\bar{\psi}=\hat{U} \psi=e^{-i T \hat{p}^{2} / 2} e^{-i k \cos \hat{x}} \psi,
$$

where the momentum $p$ is measured in recoil units, and $T$ plays the role of an effective Planck constant. The momentum operator $\hat{p}=-i \partial / \partial x$ has eigenvalues $p=n+\beta$, where $n$ is an integer and $\beta$ is the quasimomentum for a wave propagating in the $x$ direction. The particle energy is $E=E_{r} p^{2} / 2$, where $E_{r}$ is the recoil energy. We consider noninteracting atoms. It is convenient to express the time $t$ in number of map iterations. In experiments, values of time up to $t=150$ have been achieved [16]. Also, a very narrow initial momentum distribution down to $\mathrm{rms} \sigma_{\beta} \approx 0.002$ can be reached with BEC [17]. Values as high as $k \approx 4$ 
have been realized experimentally with $T$ varying between 1 and $4 \pi$ [16].

To perform the time reversal, we first write $T$ as $T=$ $4 \pi+\epsilon$. After $t_{r}$ iterations of (2), we interchange the order of kick and free propagation, change $T$ to $T^{\prime}=4 \pi-\epsilon$ and $k$ to $-k$, and let the system evolve during another $t_{r}$ iterations. Such a modification of $T$ can be easily realized experimentally (see, e.g., [17]). The sign of $k$ can be inverted by changing the sign of the detuning between the laser and the atomic transition frequencies or through a shift of the optical lattice by half a wavelength. Then for $t>t_{r}$ the map (2) becomes $\hat{U}_{r}=e^{i k \cos \hat{x}} e^{-i T^{\prime} \hat{p}^{2} / 2}$ where the second operator acts on momentum eigenstates $|n+\beta\rangle$ as $e^{-i T^{\prime}(n+\beta)^{2} / 2}=e^{i T(n+\beta)^{2} / 2} e^{-8 i \pi \beta(n+\beta / 2)}$. Thus $\hat{U}_{r}=$ $\hat{U}^{\dagger} e^{-8 i \pi \beta(\hat{n}+\beta / 2)}$ and the components with $\beta=0$ (integer values of $p$ ) are exactly reversed, while for other small $\beta$ values the time reversal works only approximately. This determines the meaning of approximate time reversal. Another way of inverting $k$ is to use in the first and last propagation steps for $t>t_{r}$ the value $T^{\prime \prime}=2 \pi-\epsilon$ instead of $T^{\prime}$. Indeed, for $\beta=0$ we have $e^{i \pi \hat{n}^{2}} e^{-i k \cos \hat{x}} e^{i \pi \hat{n}^{2}}=$ $e^{i k \cos \hat{x}}$, and thus inversion of $k$ works again exactly for $\beta=$ 0 and approximately for small $\beta$ values. In the following, we use the first method of $k$ inversion, but we checked that the second method gives similar results.

To characterize the wave packet dynamics, we use the inverse participation ratio (IPR) defined by $\xi_{t}=$ $\sum_{p} W_{p}(t) / \sum_{p} W_{p}^{2}(t)$, where $W_{p}(t)=|\langle p \mid \psi(t)\rangle|^{2}$ is the probability in momentum space at time $t$. Another useful quantity is the probability at zero momentum $W_{t}=W_{0}(t)$. For numerical simulations we used up to $N=2^{23}$ discretized momentum states with $\Delta p=1 / 40000$. Both quantities $\xi_{t}$ and $W_{t}$ are rescaled by their values at $t=0$ that makes them independent of $\Delta p$. Their dependence on time is shown in Fig. 1 which clearly demonstrates time reversal of both quantities. The return for $\xi_{t}$ is not perfect due to the contribution of nonzero quasimomentum components,

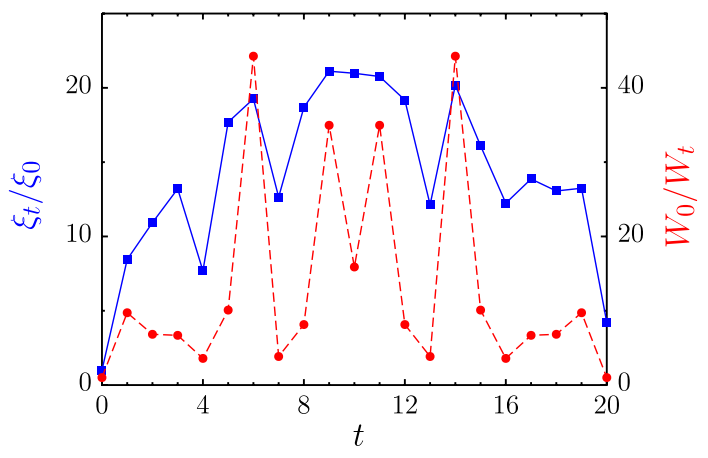

FIG. 1 (color online). Dependence on time $t$ of the rescaled IPR $\xi_{t} / \xi_{0}$ (solid curve with blue/black squares) and of the rescaled inverse probability at zero momentum $W_{0} / W_{t}$ (dashed curve with red/gray dots). Initial state is a Gaussian wave packet with $\mathrm{rms} \sigma_{\beta}=0.002$ and $k_{B} T_{0} / E_{r}=\sigma_{\beta}^{2} / 2=2 \times 10^{-6}$ (see Fig. 2). Here $k=4.5, T=4 \pi+\epsilon$ for $0 \leq t \leq t_{r}$, and $T^{\prime}=$ $4 \pi-\epsilon$ for $t_{r}<t \leq 2 t_{r}$ with $\epsilon=2$ and $t_{r}=10$. whereas the curve for $W_{t}$ is perfectly symmetric. We note that $\xi_{t}$ shows a diffusive growth for $t<4$ followed by a saturation due to dynamical localization $[6,7,12,13]$ for $t \leq t_{r}$

The momentum probability distributions at initial $t=0$ and final $t=t_{f}=2 t_{r}$ times are shown in Fig. 2. The striking feature is that the final distribution in $-0.5<p<$ 0.5 is much more narrow than the initial one and has the same maximal value since the probability at $\beta=0$ returns exactly. The shape of the reversed peak is independent of $T_{0}$. This can be interpreted as a cooling of the atoms which remained in this momentum range that defines the Loschmidt cooling mechanism. The narrowing of the central peak means that in compensation a significant fraction of atoms has obtained higher momentum values $p$ as is clearly seen in the right inset of Fig. 2. But even if the full distribution is rather broad the reversed peak is clearly dominant. On the contrary, the left inset showing the distribution at $t=t_{r}$ displays homogeneous chaotic distribution of momentum components. Thus it is the time reversal which produces the peak at the origin and performs effective cooling. It is natural to define the size of the return Loschmidt peak by its half width $\beta_{L}$ with $W_{\beta_{L}}\left(2 t_{r}\right)=W_{\beta=0}\left(2 t_{r}\right) / 2$. The fraction of returned atoms is $P_{\beta}=\sum_{-2 \beta_{L}}^{2 \beta_{L}} W_{\beta}\left(2 t_{r}\right)$ and their temperature is $T_{f}=$ $\sum_{-2 \beta_{L}}^{2 \beta_{L}} \beta^{2} W_{\beta}\left(2 t_{r}\right) /\left(2 P_{\beta}\right)$. Similarly to the case of chaotic acoustic cavities [10], quantum chaos makes the time reversed peak more visible. From an experimental viewpoint, the atoms outside the reversed peak can be eliminated by an escape process while those inside can be kept by switching on a suitable trap potential or attraction between atoms (e.g., Feschbach resonance). Such a procedure is similar to the process of evaporative cooling.

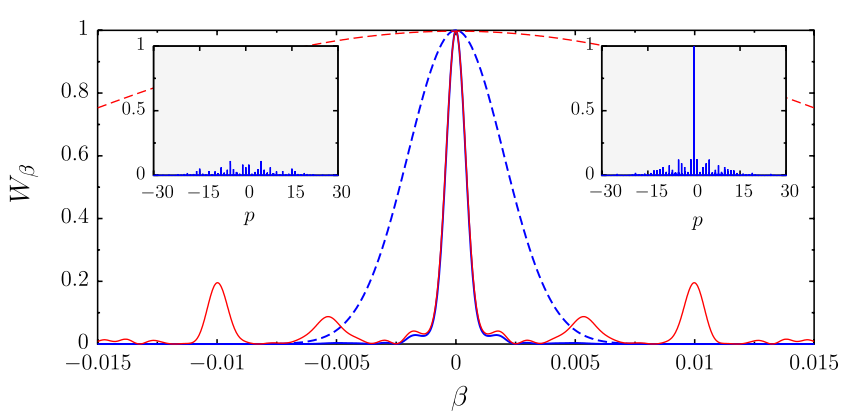

FIG. 2 (color online). Initial states probability (dashed curves) and final return probability (solid curves) shown as a function of quasimomentum $\beta$. Here $k_{B} T_{0} / E_{r}=2 \times 10^{-4}$ (red/gray curves) and $k_{B} T_{0} / E_{r}=2 \times 10^{-6}$ (blue/black curves), the latter corresponding to data of Fig. 1 and being similar to experimental conditions [17]. For return probabilities, the central peaks coincide for the two values of $T_{0}$. Insets: probability $W_{p}$ at $t=t_{r}=$ 10 (left) and final probability $W_{p}$ at $t=t_{f}=2 t_{r}$ (right) on a larger scale with $p=n+\beta$ for $k_{B} T_{0} / E_{r}=2 \times 10^{-6}$. All distributions are scaled by the value of the initial probability at zero momentum $(\beta=0)$. Parameters are as in Fig. 1. 
However, in our case the effective evaporation takes place very rapidly due to dynamical chaos.

The variation of the final return probability distribution in energy $E=E_{r} p^{2} / 2$ with $k$ is shown in Fig. 3. When $k$ increases the distribution in energy becomes more and more narrow, so that Loschmidt cooling becomes more efficient. This is related to the fact that the dynamics becomes more chaotic as $k$ increases. The temperature $T_{f}$ drops by almost 2 orders of magnitude, showing significant oscillations with $k$.

The decrease of $T_{f} / T_{0}$ with $k$ is shown in more detail in Fig. 4. It is related to the increase of the localization length $l$ of quasienergy eigenstates with $k$. Indeed, it is known that $l=D_{q} / 2$ where $D_{q}=k^{2} g\left(K_{q}\right) / 2$ is the quantum diffusion rate, $K_{q}=2 k \sin (T / 2)$ being the quantum chaos parameter [6,22]. The function $g\left(K_{q}\right)$ takes into account the effects of quantum correlations and is given [22] by $g\left(K_{q}\right)=0$ for $K_{q}<K_{c}, g\left(K_{q}\right) \approx 0.42\left(K_{q}-K_{c}\right)^{3} / K_{q}^{2}$ for $K_{c} \leq K_{q}<$ 4.5 , and $g\left(K_{q}\right)=1-2 J_{2}\left(K_{q}\right)-2 J_{1}^{2}\left(K_{q}\right)+2 J_{2}^{2}\left(K_{q}\right)+$ $2 J_{3}^{2}\left(K_{q}\right)$ for $K_{q} \geq 4.5$ where $J_{m}$ are Bessel functions. Because of this localization there is always a residual probability $W_{\beta} \sim 1 / l \sigma_{\beta}$ in the interval $-0.5<p<0.5$, even in absence of time reversal. However, this residual probability is much smaller than the maximum of the reversed peak $W_{0} \sim 1 / \sigma_{\beta}$. The width of this peak can be estimated as follows: $\beta>0$ in $\hat{U}_{r}$ acts as a small perturbation of the exactly reversible operator, whose eigenstates have $M \sim l$ components. This perturbation gives after time $t_{r}$ an accumulated quantum phase shift in the wave function $\Delta \phi=8 \pi \beta n t_{r} \approx 8 \pi \beta M t_{r}$. Thus only the atoms with $\beta \leq \beta_{L} \sim 1 /\left(8 \pi M t_{r}\right)$ return to their initial state, and their fraction is $P_{\beta} \sim \beta_{L} / \sigma_{\beta}$. The Loschmidt temperature of these atoms is $k_{B} T_{L}=E_{r} \beta_{L}^{2} / 2 \approx E_{r} /\left(128 \pi^{2} C D_{q}^{2} t_{r}^{2}+1\right)$ where $C$ is a numerical constant which according to our

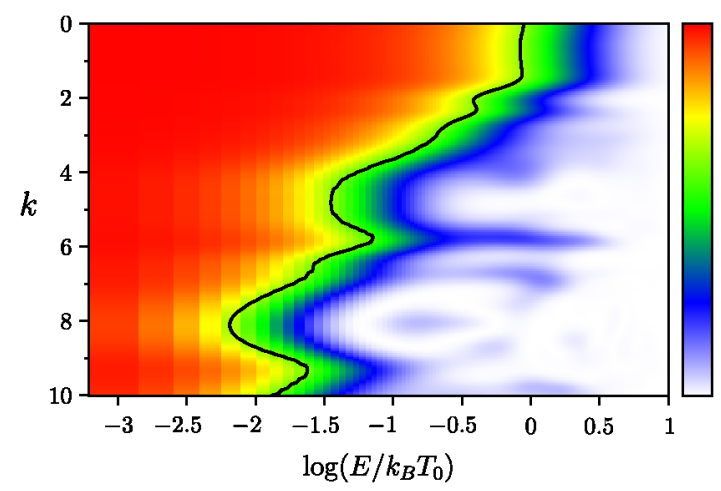

FIG. 3 (color online). Density plot of the return probability distribution $W_{p}\left(2 t_{r}\right)$ as a function of the rescaled atom energy $E / k_{B} T_{0}$ and of the kick strength $k$, where $E=E_{r} p^{2} / 2$ and $k_{B} T_{0} / E_{r}=2 \times 10^{-6}$; here $\epsilon=2$ and $t_{r}=10$. Colors denote density from white (minimal) at the right to red/gray (maximal) at the left. Black curve shows the final temperature $T_{f}$ of the return Loschmidt peak $\left(E \rightarrow k_{B} T_{f}=\langle E\rangle\right)$ as a function of $k$. Logarithm is decimal. data is $C \approx 0.4$. Thus the ratio $T_{f} / T_{0}$ is

$$
T_{f} / T_{0}=T_{L} /\left(T_{0}+T_{L}\right), \quad k_{B} T_{L} \approx E_{r} /\left(500 D_{q}^{2} t_{r}^{2}+1\right) .
$$

The formula (3) interpolates between the weakly perturbative regime with $l \ll 1$ and the strong chaos regime with $l \gg 1$. This theory assumes that $k_{B} T_{0} \ll E_{r}$ and that the localization time scale $t^{*} \approx D_{q}$ is shorter than $t_{r}$ which is approximately satisfied for most $k$ values in Fig. 4 . The theory (3) satisfactorily describes the global behavior of $T_{f} / T_{0}$ as can be seen in Fig. 4. Small scale oscillations should be attributed to mesoscopic fluctuations. These fluctuations are stronger when $\epsilon$ is varied (see inset of Fig. 4), that should be attributed to high order quantum resonances [21] at rational values of $T / 4 \pi$ [23]. We checked that the cooling remains robust even in presence of $1 \%$ fluctuations of $\epsilon$ during map iterations. For the case $t_{r} \ll t^{*}$ one should use $M \approx \sqrt{D_{q} t_{r}}$ instead of $M=D_{q}$ since the diffusion takes place during the whole time interval $t_{r}$. In this case, $k_{B} T_{L} \approx E_{r} /\left(128 \pi^{2} C D_{q} t_{r}^{3}+1\right)$.

It is important to evaluate the fraction $P_{\beta}$ of returning atoms. The estimates given above lead to $P_{\beta} \sim \beta_{L} / \sigma_{\beta} \propto$ $1 /\left(\sigma_{\beta} D_{q} t_{r}\right)$ and thus:

$$
T_{f} / T_{0} \approx P_{\beta}^{2},
$$

that is well confirmed by the data in Fig. 4. The formula (4) is written for dimension $d=1$. For higher dimensions it generalizes to $T_{f} / T_{0} \approx P_{\beta}^{2 / d}$. The data displayed in Fig. 5 show that $P_{\beta}$ drops approximately as $1 / t_{r}$, in agreement with the estimate above. For example, the cooling by a

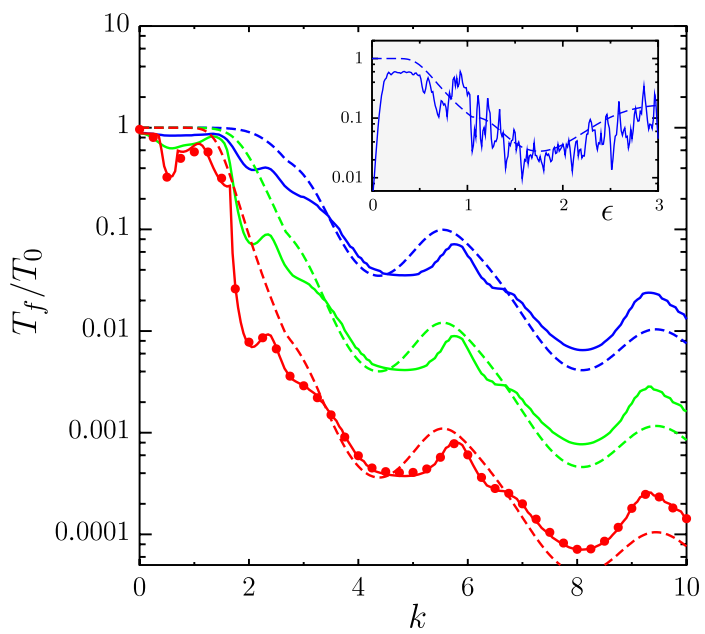

FIG. 4 (color online). Ratio of final to initial temperatures (solid curves) as a function of $k$, from top to bottom for $k_{B} T_{0} / E_{r}=2 \times 10^{-6} \quad$ (blue/black), $\quad k_{B} T_{0} / E_{r}=1.8 \times 10^{-5}$ (green/light gray), and $k_{B} T_{0} / E_{r}=2 \times 10^{-4}$ (red/gray), with $\epsilon=2$ and $t_{r}=10$. Dashed curves show the theory (3). show $P_{\beta}^{2}$ for $k_{B} T_{0} / E_{r}=2 \times 10^{-4}$, confirming theory (4). Inset: same ratio as a function of $\epsilon$ for $k=4.5$; solid curve shows numerical data and dashed curve is the theory (3). 


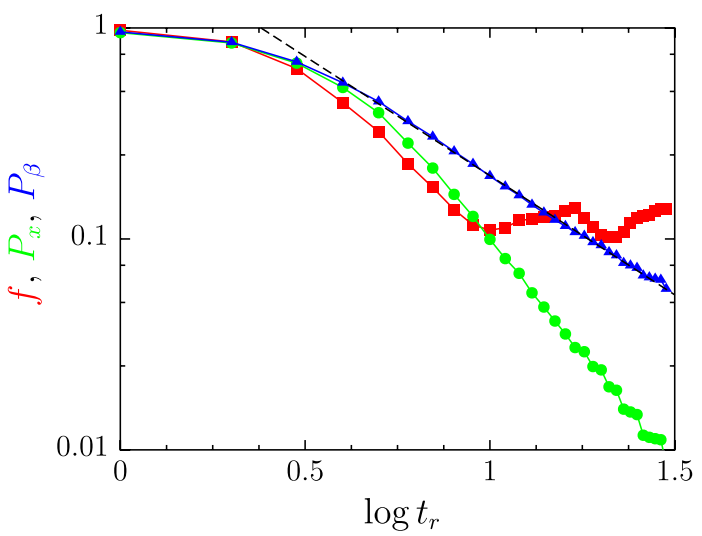

FIG. 5 (color online). Fidelity $f(\boldsymbol{\square})$, fraction $P_{\beta}$ of atoms in the Loschmidt peak ( $\boldsymbol{\Delta})$, and fraction $P_{x}$ of these atoms in the coordinate space cell $(\mathbf{O})$ (see text) as a function of time reversal moment $t_{r}$. Straight dashed curve shows the fit $\log P_{\beta}=$ $-1.13 \log t_{r}+0.43$. Parameters are as in Fig. 1. Logarithms are decimal.

factor of 100 is reached for $10 \%$ of atoms. We also checked that a significant fraction of the atoms returns not only in momentum space but also in coordinate space. To this end we define the fraction of atoms $P_{x}$ which are in the return Loschmidt peak and in the coordinate space interval $\left[-2 / \sigma_{\beta}, 2 / \sigma_{\beta}\right]$. The data show that $P_{x}$ is close to the value of $P_{\beta}$ for moderate values of $t_{r} \leq 10$, so that the Loschmidt cooled atoms remain close both in momentum and coordinate space and thus can be efficiently captured by a trap potential or a Feschbach resonance. We note that at large $t_{r}$ values $P_{\beta}$ can become smaller than the fidelity $f=\left|\left\langle\psi(0) \mid \psi\left(2 t_{r}\right)\right\rangle\right|^{2}$, which characterizes proximity of the whole wave functions and not only the reversed peak.

In conclusion, we have presented a concrete experimental proposal of time reversal of matter waves of ultracold atoms in the regime of quantum chaos. If the atoms were classical particles, they would never return back due to exponential instability of dynamical chaos. But the quantum dynamics is stable and thus a large fraction of the atoms returns back even if the time reversal is not perfect. This fraction of the atoms exhibits Loschmidt cooling which can decrease their temperature by several orders of magnitude. The reversed peak is very sensitive to variations of $\beta$ and other parameters breaking time reversal symmetry, and therefore this setup can be used as a sensitive Loschmidt interferometer to explore such a symmetry breaking (e.g., a gravitational field component along the optical lattice gives a shift in $\beta$ which affects the Loschmidt peak). The parameters considered here are well accessible to nowadays experimental setups (see, e.g., [13-20]). The realization of our proposal will shed a new light on the long-standing Boltzmann-Loschmidt dispute on time reversibility.
We thank CalMiP for access to their supercomputers and the French ANR (project INFOSYSQQ) and the EC project EuroSQIP for support.

[1] J. Loschmidt, Sitzungsberichte der Akademie der Wissenschaften, Wien, II 73, 128 (1876).

[2] L. Boltzmann, Sitzungsberichte der Akademie der Wissenschaften, Wien, II 75, 67 (1877).

[3] I. P. Kornfeld, S. V. Fomin, and Ya. G. Sinai, Ergodic Theory (Springer, New York, 1982).

[4] B. V. Chirikov, Phys. Rep. 52, 263 (1979).

[5] A. Lichtenberg and M. Lieberman, Regular and Chaotic Dynamics (Springer, New York, 1992).

[6] B. V. Chirikov, F. M. Izrailev, and D. L. Shepelyansky, Sov. Sci. Rev., Sect. C 2, 209 (1981); Physica (Amsterdam) 33D, 77 (1988).

[7] D. L. Shepelyansky, Physica (Amsterdam) 8D, 208 (1983).

[8] T. Gorin, T. Prosen, T.H. Seligman, and M. Znidaric, Phys. Rep. 435, 33 (2006).

[9] Pulsed Magnetic Resonance: NMR, ESR, and Optics: A Recognition of E. L. Hahn, edited by D. M.S. Bagguley (Oxford University Press, New York, 1992).

[10] A. Derode, P. Roux, and M. Fink, Phys. Rev. Lett. 75, 4206 (1995); J. de Rosny, A. Tourin, and M. Fink, ibid. 84, 1693 (2000).

[11] G. Lerosey, J. de Rosny, A. Tourin, A. Derode, G. Montaldo, and M. Fink, Phys. Rev. Lett. 92, 193904 (2004); G. Lerosey, J. de Rosny, A. Tourin, and M. Fink, Science 315, 1120 (2007).

[12] S. Fishman, in Quantum Chaos: E. Fermi School Course CXIX, edited by G. Casati, I. Guarneri, and U. Smilansky (North-Holland, Amsterdam, 1993), p. 187.

[13] F. L. Moore, J. C. Robinson, C. F. Bharucha, B. Sundaram, and M. G. Raizen, Phys. Rev. Lett. 75, 4598 (1995).

[14] H. Ammann, R. Gray, I. Shvarchuck, and N. Christensen, Phys. Rev. Lett. 80, 4111 (1998).

[15] S. Schlunk, M.B. d'Arcy, S. A. Gardiner, and G. S. Summy, Phys. Rev. Lett. 90, 124102 (2003).

[16] J. Chabé, G. Lemarié, B. Grémaud, D. Delande, P. Szriftgiser, and J. C. Garreau, arXiv:0709.4320.

[17] C. Ryu, M.F. Andersen, A. Vaziri, M. B. d'Arcy, J. M. Grossman, K. Helmerson, and W. D. Phillips, Phys. Rev. Lett. 96, 160403 (2006).

[18] G. Behinaein, V. Ramareddy, P. Ahmadi, and G.S. Summy, Phys. Rev. Lett. 97, 244101 (2006).

[19] J.F. Kanem, S. Maneshi, M. Partlow, M. Spanner, and A. M. Steinberg, Phys. Rev. Lett. 98, 083004 (2007).

[20] M. Sadgrove, M. Horikoshi, T. Sekimura, and K. Nakagawa, Phys. Rev. Lett. 99, 043002 (2007).

[21] F. M. Izrailev and D. L. Shepelyanskii, Theor. Math. Phys. 43, 553 (1980).

[22] D. L. Shepelyansky, Physica (Amsterdam) 28D, 103 (1987).

[23] At $\epsilon=0$ there is also a significant cooling related to ballistic propagation at the main quantum resonance; however, this effect takes place only in a very narrow $\epsilon$ range making it rather sensitive to perturbations. 